\title{
Quantum database search can do without sorting
}

\author{
Apoorva Patel* \\ CTS and SERC, Indian Institute of Science, Bangalore 560012, India
}

\begin{abstract}
Sorting is a fundamental computational process that facilitates subsequent searching of a database. It can be thought of as factorization of the search process. The location of a desired item in a sorted database can be found by classical queries that inspect one letter of the label at a time. For an unsorted database, no such classical quick search algorithm is available. If the database permits quantum queries, however, then mere digitization is sufficient for efficient search. Sorting becomes redundant with the quantum superposition of states. A quantum algorithm is written down that locates the desired item in an unsorted database a factor of two faster than the best classical algorithm can in a sorted database. This algorithm has close resemblance to the assembly process in DNA replication.
\end{abstract}

Consider a collection of items in a database, characterized by some property with values on the real line. Sorting these items means arranging them in an ordered sequence according to their property values. Though property values along the real line are sufficient for establishing the sequence, the values are taken to be distinct, and without loss of generality, they can be replaced by integer labels. The integer labels can be easily digitized, i.e., written as a string of letters belonging to a finite alphabet. When the alphabet has $a$ letters, a string of $n$ letters can label $N=a^{n}$ items. (If the number of items in the database is not a power of $a$, then the database is padded up with extra labels to make $N=a^{n}$.) In digital computers, this finite alphabet has the smallest size, i.e., $a=2$, and the letters are called bits.

The purpose of sorting is to facilitate subsequent searches of that database for items with known values of the property. Though digitization does not change the order of the items in the sequence, it simplifies the search process. One does not look for the complete string of letters in one go, but sequentially looks for one letter at a time. A dictionary is a simple example of a sorted database with alphabetical ordering. With digitization, the individual search steps have to distinguish among only a limited number of possibilities, and the maximum simplification of the search steps occurs when the alphabet has the smallest size, i.e., $a=2$. For a database of size $N$, sorting requires $O\left(N \log _{2} N\right)$ operations [1]. Once a database is sorted, all subsequent searches to locate any item in it require only $O\left(\log _{2} N\right)$ queries. Search for a desired item in an unsorted database would take $O(N / 2)$ queries, provided that the search process has a memory so that an item rejected once is not picked up again for inspection. This reduction in the search effort is what makes the laborious process of sorting worthwhile, to be carried out once and for all.

The speedup achieved by sorting can be understood as factorization of the search process. An alphabet with a finite number of letters can be looked upon as a group of finite order, and any function defined on such a group is expressible as a polynomial. A function defined with multiple letters

\footnotetext{
*Email address: adpatel@cts.iisc.ernet.in
}

becomes a multivariable polynomial. A single-variable polynomial can always be fully factored over the domain of complex numbers, but a multivariable polynomial may not be. When a polynomial can be factored, it is much more efficient to evaluate it by multiplying all the factors together than by calculating it term by term. The search function is such that it can be fully factored, resulting in a speed up of $O\left(N / \log _{2} N\right)$. As a simple case, consider a database of $N$ $=2^{n}$ items. Suppose the desired item has the label

$$
x \equiv x_{1} x_{2} x_{3} x_{4} \ldots x_{n}=1011 \ldots 0 .
$$

Any function of $x$ reduces to a polynomial in $x_{i}$ because $x_{i}$ $\in\{0,1\}$ implies $x_{i}^{2}=x_{i}$. The search process is equivalent to finding $x$ such that

$$
f(x)=\prod_{i} f_{i}\left(x_{i}\right)=x_{1}\left(1-x_{2}\right) x_{3} x_{4} \cdots\left(1-x_{n}\right)
$$

equals one. $f(x)$ evaluates to zero for all the other items in the database. In the sorted and digitized database, one searches for an item by inspecting only one $x_{i}$ at a time, i.e., evaluating $f(x)$ by sequentially combining its factors $f_{i}\left(x_{i}\right)$.

In the language of algorithms, the function $f(x)$ is referred to as an oracle. The capability of evaluating $f(x)$ for any $x$ is assigned to a black box, and the efficiency of algorithms is judged by the number of times the oracle is queried. To take advantage of a sorted database, it is crucial to have the factorized oracles, $f_{i}\left(x_{i}\right)$, available. If only the oracle $f(x)$ is available, then the search process requires $O(N / 2)$ queries even with a sorted database. The collection of factorized oracles $f_{i}\left(x_{i}\right)$ is more powerful than the global oracle $f(x)$ - one can construct $f(x)$ by combining all the $f_{i}\left(x_{i}\right)$ together, but the individual $f_{i}\left(x_{i}\right)$ cannot be determined given only $f(x)$. Generally, how an oracle is physically implemented is not of concern in the complexity analysis of an algorithm. But it is clear that $f_{i}\left(x_{i}\right)$ are easier to physically implement than $f(x)$, because $x_{i}$ span a much smaller range of values than $x$.

With a single query, the factorized oracles are able to characterize a group of items (e.g., all the items with one as the first bit of the label), unlike the global oracle that can 
characterize only a single item. With a sorted database, one takes advantage of this property of factorized oracles. The items that are addressed together by factorized oracles are arranged together in a sorted database, and not left scattered all over the place. The factorized oracles then easily pick up only the relevant group of items, and implement the search process one letter at a time, whereby the size of the group reduces in a geometric progression. If the items are not grouped together and left scattered all over, the factorized oracles do not offer any advantage compared to the global oracle.

Classically, the only way to group items together is to arrange them close to each other, as in a sorted database. Quantum physics provides another mechanism for grouping items together-quantum superposition of states. Superposed states are grouped together in the sense that they all correspond to the same physical location (in space as well as in time), and they all can be simultaneously acted upon by a single physical transformation. In a quantum search process, the inspection of items need not be carried out picking one item at a time from the database; instead, the search oracles can be applied directly to groups of items superposed together. In what follows, I demonstrate that when factorized oracles are available, quantum superposition can be used to make sorting redundant.

The quantum parallelism strategy, based on superposition of states, is quite general. It makes the algorithms more efficient, and it has been exploited to convert classically superpolynomial problems into quantum polynomial ones. Because of the linearity of quantum mechanics, when the initial state is a quantum superposition of states, the final state after carrying out the algorithmic operations will be a quantum superposition of the corresponding outcomes. Of course, at the end, quantum measurement does not permit individual determination of each outcome. But if only a single property of the possible outcomes is desired, then it may be possible to pick it up by a cleverly designed measurement (perhaps involving interference of the possible outcomes). The net result is a substantial speed up of the algorithm.

Quantum algorithms need to obey an important practical constraint, i.e., all their communications with the outside world must have classical representations. These communications include input, output, and oracles. This constraint arises from our own limitations as classical observers. Our problems are defined in a classical language, and we do not know how to make sense out of them otherwise. So we always have to find ways to extract classical meanings from quantum superpositions.

To take maximal advantage of quantum parallelism, many quantum algorithms start with a uniform quantum superposition of all possible classical input states. Such a state can be easily created from a single classical state by a fully factorized operation. For instance, each qubit in an $n$-qubit string can be put into an equal superposition of its two eigenstates, by starting with a zero string and applying the WalshHadamard transformation

$$
H=\frac{1}{\sqrt{2}}\left(\begin{array}{cc}
1 & 1 \\
1 & -1
\end{array}\right), \quad H|0\rangle=\frac{1}{\sqrt{2}}(|0\rangle+|1\rangle),
$$

$$
|00 \ldots 0\rangle \rightarrow \prod_{i=1}^{n}(H|0\rangle)_{i}=\sum_{j=1}^{N} \frac{1}{\sqrt{N}}|j\rangle .
$$

Digitization of the state labels is implicit but essential in this process. It reduces the spatial resources needed to solve the problem, i.e., $2^{n}$ states are encoded using only $n$ qubits. An obvious feature of superposition is that it is commutativethe order in which the states are superposed is irrelevant. This is the property that makes sorting redundant whenever the states are combined in a superposition.

Grover's quantum database search algorithm [2] starts with a maximally superposed state, uses a binary search function, and reaches a single state at the end. Thus, the input, the output, and the oracle have classical representations as desired. Grover's algorithm is directly applied to an unsorted database. The unitary quantum operators used are the search function $F$ (which evaluates to -1 when the labels match and to +1 when they do not) and an operation $R$ that reflects all the amplitudes about their average. Alternate application of these two operators takes the maximally superposed state to the desired state.

$$
|x\rangle=(R F) \ldots \ldots(R F)\left(\sum_{j=1}^{N} \frac{1}{\sqrt{N}}|j\rangle\right),
$$

where (RF) is repeated $O(\sqrt{N})$ times. Compared to a classical unsorted database search, quantum parallelism provides a quadratic speed up and the desired item is located with $O(\sqrt{N})$ queries. This rate, however, is much slower than that for the classical sorted database search, and we can look for ways to boost it.

Though sorting has become redundant, Grover's algorithm can still be factorized. Factorization of the search function requires digitization of the database, so let us start with a digitized database. (There is no hindrance to digitizing a database, even if it is unsorted. All that is required is that the items be distinct from each other.) Digitization makes it possible to look at one letter of the label at a time. The search process will inspect each letter of the label in turn, and decide whether it matches the corresponding letter of the desired string or not. Since Grover's algorithm requires only one query to pick one item out of four with certainty, it is efficient to digitize the quantum database with $a=4$. At the $i$ th step of the algorithm, let $F_{i}$ be the single-letter search function which looks at the $i$ th letter of the label. $F_{i}$ will evaluate to -1 when the letters match and to +1 when they do not.

$$
F_{i}=I_{1} \otimes \cdots \otimes\{ \pm 1\}_{i} \otimes \cdots \otimes I_{n} .
$$

The reflection operation $R_{i}$ that goes along with $F_{i}$ can also be written in a factorized form.

$$
R_{i}=I_{1} \otimes \cdots \otimes \frac{1}{2}\left(\begin{array}{llll}
-1 & +1 & +1 & +1 \\
+1 & -1 & +1 & +1 \\
+1 & +1 & -1 & +1 \\
+1 & +1 & +1 & -1
\end{array}\right)_{i} \otimes \cdots \otimes I_{n}
$$


When $R_{i}$ follows $F_{i}$, it carries out the same task as $R$, i.e., reflects all the amplitudes about their average. Since four is a power of two, the nontrivial transformation matrix in $R_{i}$ can be decomposed in qubit notation as $(H \otimes H) F_{0}(H \otimes H)$, where $F_{0}$ and $H$ follow a common qubit basis convention and $F_{0}$ flips the sign of the state labeled 0 . It is not mandatory, however, that $R_{i}$ be implemented in this manner.

In addition, we require the projection/measurement operators $P_{i}$, for every letter of the label. Once the $i$ th letter gets fixed by $P_{i}$, it cannot change during the subsequent steps of the algorithm, i.e., $P_{i}$ eliminates from the quantum Hilbert space all the states whose $i$ th letter does not match with the desired label. Then the factorized quantum search algorithm is

$$
|x\rangle=\prod_{i=1}^{n}\left(P_{i} R_{i} F_{i}\right)\left(\sum_{j=1}^{N} \frac{1}{\sqrt{N}}|j\rangle\right) .
$$

The starting point is the uniform superposition of all the $N$ states. Application of $R_{1} F_{1}$ doubles the amplitudes of all those strings whose first letter matches with the first letter of the desired item label, and reduces all the other amplitudes to zero. The projection/measurement operator $P_{1}$ does not change any of the amplitudes, but removes from the Hilbert space all the states with zero amplitude. The intermediate result is then a uniform superposition over the reduced Hilbert space of dimension $N / 4$, containing all the states whose first letter matches with the desired item label. One by one, the algorithm goes through all the letters of the string and ultimately finds the item with the desired label.

In the classical search of a sorted database, the sequence of $i$ in which the oracles have to be queried is determined by the convention used for grouping the items together during the sorting process. For example, with the conventional labeling as in a dictionary, one has to first query the first letter using $f_{1}\left(x_{1}\right)$, then query the second letter using $f_{2}\left(x_{2}\right)$, and so on. For the factorized quantum algorithm, Eq. (8) above, there is no such requirement; the database is unsorted and the sequence of $i$ during the execution of the algorithm does not matter.

The use of $P_{i}$ is essential here, so that the eliminated states do not take part in the subsequent steps of the algorithm, i.e., $R_{i}$ acts only on the Hilbert space of dimension $4^{1-i} N$. In a general quantum setting, the reduction of dimension of Hilbert space using $P_{i}$ is a complicated operation. But the structure of the factorized search algorithm is such that all the states eliminated by $P_{i}$ have zero amplitudes. For this limited purpose, $P_{i}$ can be denoted using identity operators,

$$
P_{i}=I_{1} \otimes \cdots \otimes\left(I_{i}\right)_{\mathrm{fix}} \otimes \cdots \otimes I_{n} .
$$

Introduction of $P_{i}$ also provides an error correcting mechanism. If the measured letter of the label does not match with the desired letter, due to imperfect implementation of $F_{i}$ and $R_{i}$ or due to effects of decoherence, then the algorithm can be restarted at the $i$ th step, with a uniform superposition of $4{ }^{1-i} N$ states. (Such a state can be prepared by setting ( $i$ $-1)$ letters to their correctly measured values, the remaining $(n-i+1)$ letters to zero, and then applying WalshHadamard transformation to these $(n-i+1)$ letters.)

The transformations $F_{i}, R_{i}$, and $P_{i}$ act on a single letter of the label only. The equations above show that the nontrivial operations are performed only on the $i$ th letter, while the rest of the letters remain unchanged (identity operations). Clearly, the resources required to implement such operations depend only on the size of the alphabet $a$, and are independent of the length of the label $n$. The physical resources needed to implement the factorized quantum search algorithm-number of letters, number of queries, and number of algorithmic steps-are therefore all proportional to $n$.

We have thus arrived at the first important result of this report: The factorized quantum search algorithm locates the desired item in an unsorted database using $O\left(\log _{4} N\right)$ queries, which is a factor of two improvement over the best search algorithm for a classical sorted database. (If the algorithm has to be restarted to correct errors, as mentioned above, then the improvement factor will be smaller.)

The frequent intervention of projection/measurement operators in this factorized algorithm makes it look like a quantum process that is stabilized by intermediate classical states. Indeed, we can think of a process closely related to searchassembling a desired string by putting together a sequence of letters. The letters have to be available in a jumbled up database and an oracle has to exist dictating the order in which the letters are to be put together. The algorithm will then carry out the assembly-line operation by adding one letter at a time. At each step, one out of $a$ items is selected, and the full string is constructed by putting together $n$ items. The quantum assembly algorithm requires a superposition of only $a$ states at each step, and not $N$ states as in Grover's algorithm; this reduction in the number of superposed states should make it easier to implement in reality. The physical resources necessary to implement the quantum assembly algorithm are again proportional to $n$, and in terms of the number of queries made, the quantum assembly algorithm provides a factor of two speed-up compared to the best classical assembly algorithm.

It is too tempting to overlook a fundamental biological process that works in this manner, i.e., replication of DNA. (As a matter of fact, biochemistry is full of assembly processes that synthesize desired objects out of their components by pattern recognition oracles.) The DNA alphabet has four letters, i.e. the bases $A, T, C, G$. In DNA itself, these bases are linked together as a string, but individual bases also randomly float around in the cellular environment. During replication the two strands of the DNA double helix separate. Each intact strand duplicates itself in an assembly-line operation, picking up one base at a time from the cellular environment. The intact strand is the factorized oracle, with the complementarity of the bases acting as the search function. The fact that this process takes place at the molecular scale and uses an alphabet of four letters raises a highly provocative thought. Could it be that the evolution of life sensed the advantage of a quantum algorithm, and opted to organize the genetic information in DNA using four bases? Note that clas- 
sically, just two bases (one complementary pair) are sufficient to carry the genetic information. This question has been discussed elsewhere [3].

Finally, it is worth noting that even though factorization is a classical computational procedure, when combined with quantum parallelism, it has proved to be useful in constructing efficient quantum algorithms. Under the best circumstances, factorization can provide a speedup of $O\left(N / \log _{2} N\right)$, and so can quantum parallelism. Shor's efficient quantum solution to the period-finding problem [4] combines factorization [fast Fourier transform (FFT) is the factorized version of Fourier transform] and quantum parallelism. Each provides a gain of $O\left(N / \log _{2} N\right)$, and the final algorithm requires $O\left[\left(\log _{2} N\right)^{2}\right]$ steps. In the search algorithm, gains of factor- ization (i.e., sorting) and quantum parallelism overlap. So one cannot obtain a speed up of $O\left(N / \log _{2} N\right)$ twice, and the quantum factorized algorithm provides only a factor of two speed up over the classical factorized algorithm. Yet another problem, that of finding parity of a set of bits, cannot gain anything from factorization and very little from quantum parallelism; the best quantum algorithm does not improve the classical one by much [both are $O(N)][5]$.

This work was supported in part by the Rajiv Gandhi Institute of Contemporary Studies in cooperation with the Jawaharlal Nehru Center for Advanced Scientific Research, Bangalore. I am grateful to Lov Grover and the Optical Physics Research group of Lucent Technologies for their hospitality during part of this work.
[1] D. E. Knuth, The Art of Computer Programming, Vol. 3: Sorting and Searching, (Addison-Wesley, Reading, MA, 1998), 2nd ed.

[2] L. Grover, Proceedings of the 28th Annual ACM Symposium on Theory of Computing (ACM Press, New York, 1996), p. 212.

[3] A. Patel, Proceedings of the Winter Institute on Foundations of Quantum Theory and Quantum Optics, edited by S. M. Roy
[Pramana 56, 367 (2001)].

[4] P. Shor, SIAM J. Sci. Comput. (USA) 26, 1484 (1997).

[5] E. Farhi, J. Goldstone, S. Gutman, and M. Sipser, Phys. Rev. Lett. 81, 5442 (1998); R. Beals, H. Buhrman, R. Cleve, M. Mosca, and R. de Wolf, Proceedings of the 39th Annual Symposium on Foundations of Computer Science (IEEE, Los Alamitos, CA, 1998), p. 352. 